\title{
Clinique et transmission du VIH chez les femmes : revue de la littérature
}

\author{
Laurence MEYER ${ }^{1}$
}

\begin{abstract}
Résumé
La progression spontanée de la maladie à VIH des femmes est similaire, voire plus lente, comparée à celle des hommes. L'initiation d'un traitement pourrait ainsi être retardée chez les femmes puisque celui-ci est basé sur des niveaux de CD4 et de charge virale qui sont spontanément plus favorables chez ces femmes. Cependant, la réponse au traitement viro-immunologique et clinique est similaire, voire meilleure, chez elles, en dépit des taux d'interruption plus élevés, peut-être en rapport avec des effets indésirables plus fréquents. En dépit des progrès majeurs sur l'espérance de vie, un excès de mortalité par rapport à la population générale non infectée persiste. L'excès absolu est similaire chez les hommes et chez les femmes, mais la mortalité des femmes étant plus faible en population générale que chez les hommes, l'excès relatif est plus important chez elles. Comprendre les déterminants de ces excès de mortalité et les réduire constituent un enjeu important.
\end{abstract}

Mots clés : sexe, genre, progression de la maladie à VIH, réponse au traitement antirétroviral, effets indésirables, grossesse, cancer du col, ménopause.

Les premiers cas de sida ont été décrits en 1981 aux États-Unis chez des homosexuels masculins. À ses débuts, l'épidémie à VIH a été considérée comme touchant essentiellement les hommes homosexuels, alors que la transmission hétérosexuelle était pourtant déjà une réalité dans la plupart des pays touchés par l'épidémie. Les premières cohortes de sujets atteints par le VIH/ sida, constituées dans les années 1983-1984, ont surtout recruté des hommes homosexuels ou hémophiles. Cela explique sans doute, en partie, pourquoi la maladie a été longtemps moins bien décrite chez les femmes. Celles-ci représentent pourtant environ $50 \%$ des personnes atteintes par le VIH dans le monde et plus encore dans certaines régions d'Afrique sub-saharienne. En France, $40 \%$ des nouveaux diagnostics concernent actuellement des femmes.

Dans cet article, sont examinés successivement d'éventuelles différences entre hommes et femmes sur le taux de transmission hétérosexuelle de l'infection, sur l'évolution spontanée de la maladie et l'évolution à l'ère des combinaisons puissantes

Inserm/Ined - Université Paris Sud 11, Le Kremlin Bicêtre. de traitements antirétroviraux, et les aspects spécifiques aux femmes touchant la vie reproductive.

\section{Transmission hétérosexuelle du VIH}

Plusieurs études réalisées sur la transmission hétérosexuelle avant l'ère des thérapies antirétrovirale sont montré que le taux de transmission dépendait du stade de l'immunodéficience atteint par le cas index ${ }^{2}$, en termes de stade clinique, de taux de lymphocytes CD4 ou de niveau de charge virale $[1,2]$. Un très jeune âge était également un facteur de risque de transmission et d'acquisition, de même que, bien sûr, la non-utilisation systématique de préservatif lors des relations sexuelles. Le rôle de la présence d'une infection génitale dans le risque de contamination n'est pas retrouvé de façon constante [1,2], et les études randomisées de prévention des maladies sexuellement transmissibles n'ont pas toutes montré leur efficacité dans la réduction de l'incidence du VIH. Le taux de transmission de l'homme vers la femme et de la femme vers l'homme est comparable dans des populations où le taux de circoncision masculine est faible, que ce soit par exemple dans l'étude ougandaise [2] ou dans l'étude européenne sur la transmission hétérosexuelle [1].

La circoncision masculine a été depuis longtemps retrouvée associée à un risque diminué de contamination dans des études d'observation [2,3]. Un essai randomisé en Afrique du Sud vient de prouver la capacité de la circoncision à diminuer l'incidence du VIH chez les hommes circoncis, diminution d'environ $60 \%$ [4]. Ces résultats ont été confirmés par deux autres essais récents au Kenya et en Ouganda. Cette relative protection de la circoncision pourrait expliquer que le taux de transmission dans le sens femme-homme dans des études menées aux États-Unis ait été inférieur à celui dans le sens homme-femme, car la prévalence de la circoncision y est élevée, de l'ordre de $70 \%$. II n'existe pas de donnée à l'heure actuelle pour déterminer si la circoncision masculine diminue aussi directement le risque de transmission de l'homme vers la femme. La circoncision peut bénéficier également indirectement aux femmes, en diminuant la prévalence du $\mathrm{VIH}$ dans la population des hommes circoncis. L'implantation de programmes de circoncision dans les pays à prévalence élevée

\footnotetext{
Cas index : sujet à l'origine de la contamination.
} 
du VIH doit cependant s'accompagner de renforcement des recommandations de prévention, afin d'éviter une augmentation paradoxale du risque de contamination des femmes engendrée par un abandon des autres stratégies de prévention [5].

La diffusion des thérapies antirétrovirales dans les pays industrialisés, en diminuant la charge virale dans le sang et dans les sécrétions génitales, s'est probablement accompagnée d'une diminution de la transmission hétérosexuelle au sein de couples sérodifférents: c'est ce que suggère une étude espagnole, qui a permis d'observer une diminution de l'ordre de $80 \%$ du risque de transmission depuis 1999, par rapport à la période 1991-1995 [6]. Cependant, la réduction des cas de transmission associée à la prise d'antirétroviraux pourrait être annihilée par la plus longue durée d'infectiosité, particulièrement si les comportements sexuels de prévention des personnes traitées se modifient [7]. En France, les données de la déclaration obligatoire des nouveaux diagnostics d'infection à VIH, disponibles depuis 2003, montrent, chez les sujets de nationalité étrangère, une stabilisation du nombre de nouveaux diagnostics chez les hommes et une diminution chez les femmes. Le nombre de nouveaux diagnostics portés chez des hommes et des femmes de nationalité française est stable depuis 2003 (cf l'article de Florence Lot sur la situation épidémiologique, page 7).

\section{La maladie à VIH chez les femmes}

Les comparaisons entre hommes et femmes de la progression de la maladie menées avant l'ère des multithérapies chez des sujets ayant un accès aux soins similaire nous renseignent sur l'histoire naturelle de la maladie. Les comparaisons selon le sexe menées depuis 1996, portant sur des sujets suivis ou des sujets traités, nous renseignent quant à elles sur les bénéfices respectifs apportés par les avancées thérapeutiques, les recommandations à formuler et les voies de recherche à poursuivre.

\section{Progression spontanée de la maladie}

\section{Progression clinique}

Des premières études réalisées dans les années 1990 aux ÉtatsUnis avaient suggéré une progression plus rapide de la maladie chez les femmes. En fait, il n'en est rien, et les différences observées étaient sans doute attribuables à des différences dans l'accès aux soins. La plupart des études et des collaborations menées chez des séroconverteurs ${ }^{3}$ suivis avant 1996, c'est-àdire avant l'avènement des traitement antirétroviraux hautements actifs, ont montré soit un risque de progression spontanée comparable chez les hommes et les femmes, soit une tendance,

\footnotetext{
3 Sujets à date de contamination connue et suivis dès leur séroconversion. Les études de séroconverteurs sont les seules valides, lorsque l'on veut comparer des groupes sur le risque de progression de la maladie à VIH depuis la contamination, surtout lorsque, comme c'est le cas pour les femmes comparées aux hommes, les taux de CD4 à délai égal depuis la contamination diffèrent.
}

en général de faible ampleur, vers une progression moins rapide chez les femmes [8-13]. La différence entre les sexes apparaissait un peu plus marquée pour le risque de décès que pour le risque de sida. Ces résultats ont été retrouvés récemment dans la large collaboration Cascade à laquelle participent la plupart des cohortes européennes de séroconverteurs [14].

\section{Comparaison des niveaux des marqueurs $\mathrm{CD} 4$ et charge virale}

La charge virale plasmatique et le taux de CD4 sont des marqueurs très prédictifs de la progression spontanée ultérieure de la maladie $[15,16]$, qu'ils soient mesurés chez des séroconverteurs ou chez des sujets diagnostiqués au stade chronique.

Des taux de CD4 différents entre les sexes ont été décrits chez des sujets non infectés, soit en population générale [17], soit chez des enfants non infectés nés de mères séropositives [18]. Les comparaisons femmes-hommes menées chez des séroconverteurs suivis avant 1996 dans les pays industrialisés ont retrouvé de telles différences chez les sujets infectés : les femmes avaient, en tout cas dans les premières années suivant la contamination, des taux de CD4 plus élevés que les hommes, d'environ 50 à $100 \mathrm{CD} 4 / \mathrm{ml}$ [voir revue dans 19]. Le mécanisme n'en est pas clair ; l'influence de facteurs hormonaux a bien sûr été invoquée. En effet, les femmes ménopausées, qu'elles soient infectées par le VIH ou non, ont des taux de CD4 plus bas que les femmes en âge de procréer [20,21]. Des données préliminaires récentes provenant des cohortes Anrs-Primo et Primo-Cl suggèrent cependant que l'on ne retrouve pas des taux de CD4 plus élevés chez les femmes infectées en population africaine. Cela pourrait être expliqué par des taux de CD4 d'emblée plus bas chez les Africains. Enfin, des résultats discordants existent sur la persistance éventuelle de cette différence de CD4 au cours de la progression spontanée de l'immunodéficience. En France, femmes et hommes avaient des taux de CD4 identiques au moment du diagnostic de sida clinique, avant l'avènement des traitements antirétroviraux ${ }^{4}$; ce résultat plaide plutôt en faveur d'une diminution de la différence, au fur et à mesure de l'aggravation de l'immunodéficience.

De nombreuses études, résumées dans plusieurs revues de la littérature, ont également rapporté des charges virales plus basses chez les femmes que chez les hommes [19, 22, 23]. Les écarts de charge virale, de l'ordre de 0,3 à $0,7 \log ^{5}$, paraissent d'autant moins importants que le taux de CD4 est moins élevé. Cela semble indiquer que l'écart entre les sexes diminuerait au cours de la progression de la maladie à VIH. Des différences de charge virale selon le sexe ont aussi été décrites en Afrique et en Thaillande [24-26].

\footnotetext{
Source : Florence Lot, Institut national de veille sanitaire.

En d'autres termes, si on exprime une charge virale en nombre absolu de copies plutôt qu'en log de copies : pour une valeur moyenne de 10000 copies/ml dans un groupe d'hommes, la valeur moyenne dans un groupe de femmes comparables est plus basse et peut aller de 2000 à 5000 copies $/ \mathrm{ml}$.
} 
Ces différences dans les niveaux des marqueurs CD4 et charge virale entre hommes et femmes appellent plusieurs remarques. Elles pourraient expliquer que les femmes contrôlent spontanément plus souvent leur charge virale que les hommes [27] et qu'elles gardent plus souvent que les hommes leur statut asymptomatique à long terme [28]. Ces différences, dont le mécanisme reste encore à trouver, semblent cependant avoir peu d'impact sur la progression spontanée de la maladie vers le sida et le décès, qui diffère peu entre hommes et femmes ${ }^{6}$.

\section{Progression de la maladie à l'ère des traitements antirétroviraux}

\section{Pharmacocinétique, effets secondaires}

Du point de vue pharmacocinétique, quelques études, peu nombreuses et portant sur un nombre limité de molécules (zidovudine, lamivudine, abacavir, névirapine, saquinavir), ont décrit des concentrations sanguines plus élevées chez les femmes [29-32]. Les études de pharmacocinétique sont sensibles à de multiples facteurs de variabilité, et le rôle du sexe ici pourrait s'expliquer par des différences de masse corporelle. Ces résultats peuvent expliquer que les femmes expérimentent plus souvent que les hommes certains effets indésirables liés à des nucléosidiques, des non nucléosidiques ou des inhibiteurs de protéase [33-42]. Plusieurs études multicentriques ont également montré des taux d'interruption de traitement supérieurs chez les femmes [43,44], les posologies ne tenant en général pas compte du poids, qui est en moyenne plus faible chez ces dernières.

\section{Réponse viro-immunologique et clinique au traitement}

L'initiation d'un traitement antirétroviral se fait principalement sur des seuils des marqueurs CD4 et charge virale. Si ceux-ci n'ont pas les mêmes niveaux chez les hommes et chez les femmes à délai égal depuis la contamination, les femmes pourraient débuter le traitement à un stade plus tardif que les hommes, voire trop tardif. En pratique, un faisceau d'arguments différents ne conduit pas actuellement à plaider en faveur d'une adaptation des recommandations d'initiation thérapeutique selon le sexe. D'une part, la plupart des études ont montré que la réponse viroimmunologique à court terme ne diffère pas entre hommes et femmes, de même que la réponse clinique, sida ou décès, à plus long terme [revue dans 19]. Ces résultats ont été établis sur de larges collaborations internationales, où le nombre de sujets et le nombre d'événements assurent une puissance raisonnable [45]. Par ailleurs, des analyses menées cette fois à l'échelle de la population, incluant donc à la fois des sujets traités et des sujets non traités, montrent que la progression clinique de la maladie

\footnotetext{
Elles impliquent, en revanche, que, lorsque l'on étudie la progression de la maladie chez des sujets dont on ignore la date de contamination (cas le plus fréquent dans les files actives hospitalières), il ne faut pas ajuster sur la charge virale et le taux de CD4 pour les comparaisons hommes-femmes : un tel ajustement, contre-productif, aurait comme conséquence de créer artificiellement une différence entre les deux groupes.
}

n'est pas plus rapide depuis 1996 chez les femmes que chez les hommes. Cela suggère que femmes et hommes ont bénéficié de la même façon des avancées thérapeutiques en termes de ralentissement d'évolution clinique de la maladie. Des données plus récentes issues de la collaboration Cascade ont même montré depuis 1997, chez les femmes, une diminution significative du risque d'évolution vers le sida et vers le décès [14]. On ne peut exclure que les femmes aient en moyenne une meilleure observance que les hommes. II est possible également que l'effet puissant des traitement antirétroviraux permette, sur le long terme, de retrouver ce qui est bien connu en population générale, à savoir à la fois une mortalité plus faible et une incidence plus faible de certaines pathologies telles que la tuberculose, la maladie de Kaposi ou les lymphomes [14].

\section{Comparaisons de la mortalité à celle de la population générale}

Si l'on compare maintenant non plus seulement hommes et femmes infectés entre eux, mais les taux de mortalité observés chez les personnes infectées à ceux de la population générale, les travaux des cohortes Anrs-Aproco et Aquitaine ont montré que, pour les patients mis au traitement en France entre 1997 et 1999 et suivis jusqu'en 2005, les ratios de mortalité standardisés sur l'âge étaient de 4,7 pour les hommes et de 19,5 pour les femmes [46]. En d'autres termes, si la survie des femmes infectées tendait à être meilleure que celle des hommes infectés dans les périodes les plus récentes, elle restait cependant considérablement moins bonne que celle des femmes non infectées de même âge. De même, aux Pays-Bas, les ratios de mortalité standardisés sur l'âge pour les patients ayant reçu une multithérapie entre 1996 et 2003 ont été également retrouvés plus élevés chez les femmes que chez les hommes, par rapport à la population générale néerlandaise [47]. Plusieurs précautions doivent cependant être apportées à ces résultats. Ce type de comparaison à la population générale ne peut pas tenir compte de certains facteurs, comme la co-infection à VHC, la consommation de tabac et le niveau socio-économique, dont le poids sur la mortalité est élevé7. Or les fréquences du tabagisme et de la coinfection à VHC ont été plus élevées chez les sujets infectés par le VIH qu'en population générale, même si elles sont actuellement en diminution. De plus, ces analyses comprennent pour partie des patients ayant nécessité une mise au traitement dans les années 1996-1999; ces patients avaient des facteurs pronostiques sans doute moins bons que ceux observés actuellement et ont reçu des combinaisons thérapeutiques sans doute moins optimales. Ces résultats doivent aussi s'interpréter en regard des chiffres absolus d'excès de mortalité. Ils sont de l'ordre de 2 pour 100 personnes-années, et varient peu avec l'âge et le sexe [47, 48]. La mortalité des femmes en population générale étant très

Ce type de renseignements n'est pas disponible en routine dans les statistiques de mortalité. 
inférieure à celle des hommes, une même différence absolue engendre des rapports de risque plus importants chez les femmes que chez les hommes.

\section{Événements et pathologies spécifiques des femmes}

\section{Grossesses}

Chez les femmes non infectées, la grossesse peut modifier l'évolution de certaines maladies auto-immunes. Par contre, les nombreuses études menées sur l'influence éventuelle d'une grossesse sur l'évolution clinique spontanée de la maladie à VIH chez la femme ont permis de conclure à l'absence d'effet péjoratif [49]. Ces résultats sont concordants avec ceux observés sur l'évolution des CD4 pendant la grossesse : un déclin dans le nombre de $\mathrm{CD} 4 / \mathrm{ml}$ pendant le premier trimestre, suivi d'une remontée graduelle pour atteindre les taux de départ dans les premiers mois suivant l'accouchement. En revanche, le pourcentage de CD4 est beaucoup plus stable au cours de la grossesse. Enfin, la survenue d'une grossesse ne modifie pas la dynamique d'évolution du taux de CD4 sur une période de plusieurs années chez des femmes à date de contamination connue [21]. Une étude récente menée à l'ère des traitements antirétroviraux a même montré un risque réduit de progression de la maladie chez les femmes ayant eu une grossesse par rapport aux autres [50]. Un tel résultat s'explique très probablement par un meilleur état de santé préalable à la grossesse, qui contribuerait ainsi à sa survenue.

Le déclin majeur du taux de transmission mère-enfant lié à l'administration d'antirétroviraux a probablement conduit de plus en plus de femmes infectées à souhaiter un enfant. C'est ce qui avait été observé dans les cohortes françaises Anrs-Seroco et Serogest, où le taux d'incidence des naissances tendait à augmenter dans la période 1994-1996, par rapport aux périodes antérieures [51]. Peu de recherches ont jusqu'à présent étudié l'impact de la diffusion des traitements antirétroviraux sur l'incidence des grossesses; une diminution de la proportion des avortements parmi les grossesses a été observée depuis 1996 en Europe et aux États-Unis [52, 53]. La complexité grandissante de la prise en charge thérapeutique des femmes enceintes infectées par le VIH nécessite de continuer à surveiller à la fois le taux de transmission mère-enfant et la survenue de potentiels effets néfastes chez la mère comme chez l'enfant.

\section{Aménorrhée, anovulation, ménopause}

Très peu d'études se sont jusqu'à présent intéressées aux troubles des cycles, aménorrhée, anovulation, et aux troubles de la ménopause en rapport avec l'infection à VIH [54-59]. Elles nécessitent un groupe témoin de femmes non infectées, les cycles anovulatoires étant fréquents en population générale (5 à $30 \%$ chez des femmes ayant des cycles réguliers). Ces comparaisons doivent de plus tenir compte de nombreux facteurs tels que l'index de masse corporelle, l'usage de substances illicites, et le statut socio-économique. On dispose également de très peu de données sur les relations potentielles entre les troubles du cycle menstruel chez les femmes traitées par traitements antirétroviraux et la survenue de lipodystrophies. Ces champs de recherches sont en cours d'exploration en France dans la cohorte Anrs-Copana.

\section{Pathologies du col utérin}

La relation entre papillomavirus, virus à transmission sexuelle, et lésions cervicales intra-épithéliales et cancer est maintenant bien établie. Un taux très élevé de lésions cervicales a été observé chez les femmes infectées par le VIH, comparé à celui observé chez des femmes non infectées, y compris après prise en compte du comportement sexuel (âge au premier rapport sexuel et nombre de partenaires sexuels essentiellement). L'incidence des lésions squameuses intra-épithéliales avant la période des traitement antirétroviraux était de 8,3 pour 100 personnes-années, versus 1,8 chez des femmes non infectées de même niveau socio-économique [60]. L'association est d'autant plus forte que le taux de CD4 des femmes infectées est bas. Le taux de régression est inversement proportionnel au niveau de $\mathrm{CD} 4$, mais est deux fois plus fréquent chez les femmes recevant un traitement antirétroviral $[61,62]$. À l'inverse du cancer in situ, l'incidence du cancer invasif, plus sensible à un défaut de dépistage, n'a pas été trouvée plus élevée chez les femmes infectées dans une large étude récente portant sur des femmes ayant un accès aux soins régulier et comparable à celui de femmes non infectées [63].

\section{Accès au diagnostic et à la prise en charge}

Enfin, pour conclure cet article, il faut souligner que les comparaisons femmes-hommes menées dans des études réalisées chez des sujets pris en charge à l'ère des traitements antirétroviraux ne sont généralisables que si l'accès au diagnostic et aux soins ne diffère pas trop entre hommes et femmes. En effet, s'il n'y a pas d'argument fort pour penser qu'un traitement précoce, lors de la primo-infection, peut ralentir l'évolution ultérieure de la maladie par rapport à un traitement différé $[64,65]$, il est certain, en revanche, qu'une prise en charge à un stade trop avancé de l'immunodépression est associée à une surmortalité importante dans les premiers mois suivant l'instauration d'un traitement [66]. D'après la base de données française FHDH, qui recense environ $70 \%$ des personnes infectées par le $\mathrm{VIH}$ et prises en charge à l'hôpital en France, les hommes étaient plus à risque que les femmes de se présenter tardivement au stade sida ou avec des taux de CD4 inférieurs à 200/ml [66]. De même, les données de la déclaration obligatoire des nouveaux diagnostics de séropositivité montrent que le pourcentage de femmes diagnostiquées au stade de sida clinique est inférieur au pourcentage d'hommes, qu'il s'agisse de patients français ou 
de nationalité étrangère ${ }^{8}$. Le dépistage du VIH est systématiquement proposé aux femmes enceintes en France, ce qui peut sans doute expliquer que le diagnostic soit en moyenne moins tardif chez les femmes. En revanche, le pourcentage de femmes chez les patients diagnostiqués au stade très précoce de la primoinfection est de $20 \%$ dans la cohorte Anrs-Primo, plus faible que celui de $39 \%$ observé chez les patients pris en charge au stade chronique [67]. Cette différence s'explique probablement par un accès au dépistage très précoce plus important chez les hommes homosexuels. En conclusion, s'il y a moins de dépistage tardif chez les femmes que chez les hommes, il y a également moins de dépistage très précoce, au stade de la primo-infection.

\section{Conclusion}

Dans les pays industrialisés, avant la diffusion des traitements antirétroviraux, la progression spontanée de la maladie chez les femmes était similaire à celle des hommes, voire légèrement plus favorable. La plupart des études ont relevé une charge virale plus basse et des taux de CD4 plus élevés chez les femmes que chez les hommes dans les premières années suivant la contamination. On dispose de peu d'arguments pour expliquer de telles différences, qui ne sont pas retrouvées dans toutes les populations, au moins pour ce qui concerne le taux de CD4. L'initiation d'un traitement pourrait être tardive chez les femmes, puisqu'il est basé sur le taux de CD4, qui est spontanément plus élevé chez les femmes. Les études de séroconverteurs n'ont pourtant pas montré, depuis l'avènement des traitements antirétroviraux, de progression clinique de la maladie plus défavorable chez les femmes à l'échelle de la population. Quand on considère les sujets traités, la réponse au traitement viro-immunologique ou clinique est similaire, voire meilleure, chez les femmes, malgré des taux d'interruption plus élevés, possiblement en rapport avec des survenues d'effets indésirables plus fréquents. En dépit des progrès majeurs sur l'espérance de vie réalisés depuis 1996, il persiste en France un excès de mortalité chez les personnes infectées, par rapport à la population générale non infectée. L'excès absolu est du même ordre chez les hommes et chez les femmes, mais, du fait de la faible mortalité des femmes en population générale, l'excès relatif est plus important chez celles-ci. Comprendre les déterminants de ces excès de mortalité et les réduire constituent un des enjeux importants des recherches à venir, dans les pays industrialisés comme dans les pays moins développés. Par ailleurs, avec l'allongement de l'espérance de vie apporté par les traitements antirétroviraux, des événements inattendus et des excès d'incidence de certaines pathologies peuvent apparaître. Continuer à surveiller l'incidence de tels événements au sein de cohortes d'hommes et de femmes suivis au long cours est ainsi une priorité. Enfin, les essais randomisés récents ont prouvé l'efficacité de la circoncision masculine dans

\footnotetext{
8 Source : Florence Lot, Institut national de veille sanitaire.
}

la diminution de l'incidence de l'infection à VIH chez les hommes circoncis. La capacité des programmes de circoncision masculine, qui vont être mis en place dans des pays à forte prévalence du VIH, à diminuer l'incidence de l'infection à VIH également chez les femmes est un des enjeux majeurs actuels de la prévention.

\section{Références bibliographiques}

1. Vincenzi de I. A longitudinal study of human immunodeficiency virus transmission by heterosexual partners. European study group on heterosexual transmission of HIV. N Engl J Med 1994 ; 331 : 341-6.

2. Quinn TC, Waver MJ, Sewankambo N, et al. Viral load and heterosexual transmission of human immunodeficiency syndrome virus type 1. N Engl J Med $2000 ; 342: 921-9$.

3. Vincenzi de I, Mertens T. Male circumcision : a role in HIV prevention? AIDS $1994 ; 8: 153-60$.

4. Auvert B, Taljaard D, Lagarde E, et al. Randomised controlled intervention trial of male circumcision for reduction of HIV infection risk: the Anrs 1265 trial. PLoS Med 2005; 2 : e298.

5. Hankins C. Male circumcision : implications for women as sexual partners and parents. Reproductive Health Matters 2007 ; 15 : 62-7.

6. Castilla J, del Romero J, Hernando V, Marincovich B, Garcia S, Rodriguez C. Effectiveness of highly active antiretroviral therapy in reducing heterosexual transmission of HIV. J Acquir Immune Defic Syndr 2005 ; 40 : 96-101.

7. McCormick AW, Walensky RP, Lipsitch M, et al. The effect of antiretroviral therapy on secondary transmission of HIV among men who have sex with men. Clin Inf Dis 2007 ; 44 : 1115-22.

8. Cozzi Lepri A, Pezzotti P, Dorrucci M, Phillips AN, Rezza G. HIV disease progression in 854 women and men infected through injecting drug use and heterosexual sex and followed for up to nine years from seroconversion. Italian Seroconversion Study. BMJ 1994 ; 309 : 1537-1542.

9. Brettle RP, McNeil AJ, Burns S, et al. Progression of HIV : follow-up of Edinburgh drug users with narrow seroconversion intervals in 1983-1985. AIDS 1996; $10: 419-430$.

10. Pezzotti P, Galai N, Vlahov D, Rezza G, Lyles CM, Astemborski J. Direct comparison of time to AIDS and infectious disease death between HIV seroconverter injection drug users in Italy and the United States: results from the ALIVE and ISS studies. AIDS Link to Intravenous Experiences. Italian Seroconversion Study. J Acquir Immune Defic Syndr 1999; 20 : 275-282.

11. Prins M, Robertson JR, Brettle RP, et al. Do gender differences in CD4 cell counts matter? AIDS 1999; $13: 2361-2364$

12. Collaborative Group on AIDS incubation and HIV survival including the CASCADE EU Concerted Action. Time from HIV-1 seroconversion to AIDS and death before widespread use of highly-active antiretroviral therapy: a collaborative re-analysis. Lancet $2000 ; 355: 1131-1137$.

13. Hubert JB, Rouzioux C, Boufassa F, Delfraissy JF, Meyer L. Gender, disease progression and response to HAART. [Abstract ThOrC1448] XIV International AIDS Conference, Barcelona, July 2002.

14. Jarrin I, Geskus R, Bashkaran K, et al. Changes over time in the risk of AIDS and death: slower disease progression in women from 1997 onwards. Manuscrit en revue. 
15. Mellors JW, Munoz A, Giorgi JV, et al. Plasma viral load and CD4+ lymphocytes as prognostic markers of HIV-1 infection. Ann Intern Med 1997; 126 : 946954.

16. Hubert JB, Burgard M, Dussaix E, et al. Natural history of serum HIV-1 RNA levels in 330 patients with a known date of infection. The SEROCO Study Group. AIDS $2000 ; 14: 123-131$.

17. Tollerud DJ, Clark JW, Morris Brown L, et al. The influence of age, race, and gender on peripheral blood mononuclear cell subsets in healthy non smokers. J Clin Immunol $1989 ; 9: 214-22$.

18. European Collaborative Study. Are there gender and race differences in cellular immunity patterns over age in infected and uninfected children born to HIV-infected women? J Acquir Immune Defic Syndr $2003 ; 33$ : 635-41.

19. Prins M, Meyer L, Hessol N. Sex and the course of HIV infection in the PreHAART and HAART era : A review of the literature. AIDS $2005 ; 19: 357-70$.

20. Giglio T, Imro MA, Filaci G, et al. S. Immune cell circulating subsets are affected by gonadal function. Life Sci $1994 ; 54: 1305-12$.

21. Van Benthem BH, Vernazza P, Coutinho RA, Prins M, The European study on the natural history of HIV infection in women. The impact of pregnancy and menopause on CD4 lymphocyte counts in HIV-infected women. AIDS 2002; $16: 919-924$.

22. Napravnik S, Poole C, Thomas JC, Eron JJ. Gender difference in HIV RNA levels : a meta-analysis of published studies. J Acquir Immune Defic Syndr $2002 ; 31: 11-19$.

23. Gandhi M, Bacchetti P, Miotti P, Quinn TC, Veronese F, Greenblatt RM. Does patient sex affect human immunodeficiency virus levels? Clin Infect Dis $2002 ; 35: 313-322$.

24. Morgan D, Rutebemberwa A, Malamba S, et al. HIV-1 RNA levels in an African population-based cohort and their relation to CD4 lymphocyte counts and World Health Organization clinical staging. J Acquir Immune Defic Syndr $1999 ; 22: 167-173$.

25. Trichavaroj R, de Souza MS, Buapunth P, et al. HIV viral load in Thai men and women with subtype E infections. J Acquir Immune Defic Syndr 2001; 26 : 345-347.

26. Gray RH, Li X, Wawer MJ, et al. Determinants of HIV-1 load in subjects with early and later HIV infections, in a general-population cohort of Rakai, Uganda. $J$ Infect Dis 2004 ; 189 : 1209-1215.

27. Madec $Y$, Boufassa F, Porter $K$, and Meyer $L$ on behalf of the CASCADE collaboration. Spontaneous control of viral load and CD4 progression among HIV-1 seroconverters. AIDS $2005 ; 19: 2001-7$.

28. Madec $\mathrm{Y}$, Boufassa F, Avettand-Fenoel V, et al. The Anrs-SEROCO/HEMOCO study group. Early control of HIV-1 infection in long-term non progressors (LTNPS) followed since diagnosis in the Arns SEROCO/HEMOCO Cohort. Manuscrit en revue

29. Anderson PL, Kakuda TN, Kawle S, Fletcher CV. Antiviral dynamics and sex differences of zidovudine and lamivudine triphosphate concentrations in HIVinfected individuals. AIDS 2003; $17: 2159-2168$.

30. Harris M, Back D, Kewn S, Jutha S, Marina R, Montaner JS. Intracellular carbovir triphosphate levels in patients taking abacavir once a day. AIDS $2002 ; 16: 1196-1197$.

31. Regazzi M, Villani $P$, Seminari $E$, et al. Sex differences in nevirapine disposition in HIV-infected patients. AIDS $2003 ; 17: 2399-2400$.

32. Fletcher $\mathrm{CV}$, Jiang $\mathrm{H}$, Brundage RC, et al. Sex-based differences in saquinavir pharmacology and virologic response in AIDS Clinical Trials Group Study 359. $J$ Infect Dis 2004 ; 189 : 1176-1184.
33. Currier JS, Spino C, Grimes J, et al. Differences between women and men in adverse events and $\mathrm{CD} 4+$ responses to nucleoside analogue therapy for HIV infection. The Aids Clinical Trials Group 175 Team. J Acquir Immune Defic Syndr $2000 ; 24: 316-324$.

34. Moore RD, Fortgang I, Keruly J, Chaisson RE. Adverse events from drug therapy for human immunodeficiency virus disease. Am J Med 1996; 101 :34-40.

35. Gatti G, Di Biagio A, Casazza R, et al. The relationship between ritonavir plasma levels and side-effects : implications for therapeutic drug monitoring. AIDS $1999 ; 13: 2083-2089$.

36. Bonfanti $\mathrm{P}$, Valsecchi L, Parazzini $\mathrm{F}$, et al. Incidence of adverse reactions in HIV patients treated with protease inhibitors : a cohort study. Coordinamento Italiano Studio Allergia e Infezione da HIV (CISAl) Group. J Acquir Immune Defic Syndr $2000 ; 23: 236-245$.

37. Lucas GM, Chaisson RE, Moore RD. Highly active antiretroviral therapy in a large urban clinic : risk factors for virologic failure and adverse drug reactions. Annals of Internal Medicine $1999 ; 131: 81-87$.

38. Guay LA, Musoke P, Fleming T, et al. Intrapartum and neonatal single-dose nevirapine compared with zidovudine for prevention of mother-to-child transmission of HIV-1 in Kampala, Uganda : HIVNET 012 randomised trial. Lancet $1999 ; 354: 795-802$.

39. Bersoff-Matcha SJ, Miller WC, Aberg JA, et al. Sex differences in nevirapine rash. Clin Infect Dis 2001; 32: 124-129.

40. Antinori A, Baldini F, Girardi $E$, et al. Female sex and the use of anti-allergic agents increase the risk of developing cutaneous rash associated with nevirapine therapy. AIDS $2001 ; 15: 1579-1581$.

41. Mazhude C, Jones S, Murad S, Taylor C, Easterbrook P. Female sex but not ethnicity is a strong predictor of non-nucleoside reverse transcriptase inhibitor-induced rash. AIDS 2002; 16 : 1566-68.

42. Wong KH, Chan KC, Lee SS. Sex differences in nevirapine rash. Clin Infect Dis $2001 ; 33: 2096-8$.

43. Murri R, Lepri AC, Phillips AN, et al. Access to antiretroviral treatment, incidence of sustained therapy interruptions, and risk of clinical events according to sex : evidence from the I.CoNA Study. J Acquir Immune Defic Syndr $2003 ; 34: 184-190$.

44. Touloumi G, Pantazis N, Antoniou A, Stirnadel HA, Walker SA, Porter K on behalf of the CASCADE collaboration. Highly active antiretroviral therapy interruption : predictors and virologic and immunological consequences. J Acquir Immune Defic Syndr 2006 ; 42 : 554-61.

45. Egger M, Chene G, Sterne JA, et al. Prognostic importance of initial response in HIV-1 infected patients starting potent antiretroviral therapy: analysis of prospective studies. Lancet $2003 ; 362: 679-686$.

46. Lewden C, Chene G, Morlat P, et al. HIV-infected adults with a CD4 cell count greater than $500 \mathrm{cells} / \mathrm{mm}^{3}$ on long-term combination antiretroviral therapy reach same mortality rates as the general population. J Acquir Immune Defic Syndr $2007 ; 46: 72-7$.

47. Van Sighem A, Danner S, Ghani AC, Gras L, Anderson RM, de Wolf F on behalf of the Athena National observational cohort study. Mortality in patients with successful initial response to highly active antiretroviral therapy is still higher than in non HIV-infected individuals. J Acquir Immune Defic Syndr $2005 ; 40$ : 212-8.

48. Jaggy C, von Overbeck J, Lederberger B, et al. Mortality in the Swiss HIV cohort study and the Swiss general population. Lancet $2003 ; 362: 877-8$.

49. Saada M, Le Chenadec J, Berrebi A, et al. Pregnancy and progression to AIDS : results of the French prospective cohorts. Serogest and Seroco Study Groups. AIDS $2000 ; 14: 2355-2360$. 
50. Tai JH, Udoji MA, Barkanic G, et al. Pregnancy and HIV disease progression during the era of highly active antiretroviral therapy. J Infect Dis. $2007 ; 196$ : 1044-52.

51. Fourquet F, Le Chenadec J, Mayaux MJ, Meyer L. Pregnancies in HIV-infected women living in France. AIDS $2001 ; 15: 2193-6$.

52. Van Benthem BH, De Vincenzi I, Delmas MC, Larsen C, Van den Hoek A, Prins M. Pregnancies before and after HIV diagnosis in a European cohort of HIVinfected women. European Study on the Natural History of HIV Infection in Women. AIDS $2000 ; 14: 2171-2178$.

53. Massad LS, Springer G, Jacobson L, et al. Pregnancy rates and predictors of conception, miscarriage and abortion in US women with HIV. AIDS 2004 ; $18: 281-286$

54. Harlow SD, Ephross SA. Epidemiology of menstruation and its relevance to women's health. Epidemiol Rev $1995 ; 17: 265-286$.

55. Chirgwin KD, Feldman J, Muneyyirci-Delale 0, Landesman S, Minkoff $H$. Menstrual function in human immunodeficiency virus-infected women without acquired immunodeficiency syndrome. J Acquir Immune Defic Syndr Hum Retrovirol $1996 ; 12 ; 489-494$.

56. Harlow SD, Schuman P, Cohen M, et al. Effect of HIV infection on menstrual cycle length. J Acquir Immune Defic Syndr $2000 ; 24: 68-75$.

57. Clark RA, Mulligan K, Stamenovic $E$, et al. Frequency of anovulation and early menopause among women enrolled in selected adultAIDS clinical trials group studies. J Infect Dis $2001 ; 184: 1325-1327$.

58. Reichelderfer PS, Coombs RW, Wright DJ, et al. Effect of menstrual cycle on HIV-1 levels in the peripheral blood and genital tract. WHS 001 Study Team. AIDS 2000; 14 : 2101-2107.
59. Clark RA, Cohn SE, Jarek C, et al. Perimenopausal symptomatology among HIV-infected women at least 40 years of age. J Acquir Immune Defic Syndr $2000 ; 23: 99-100$.

60. Ellerbrock TV, Chiasson MA, Bush TJ, et al. Incidence of cervical squamous intraepithelial lesions in HIV-infected women. JAMA $2000 ; 283: 1031-1037$.

61. Heard I, Tassie JM, Kazatchkine MD, Orth G. Highly active antiretroviral therapy enhances regression of cervical intraepithelial neoplasia in HIV-seropositive women. AlDS $2002 ; 16: 1799-1802$.

62. Minkoff H, Ahdieh L, Massad LS, et al. The effect of highly active antiretroviral therapy on cervical cytologic changes associated with oncogenic HPV among HIV-infected women. AIDS $2001 ; 15: 2157-2164$.

63. Massad LS, Seaberg EC, Watts DH, et al. Low incidence of invasive cervical cancer among HIV-infected US women in a prevention program. AIDS 2004; $18: 109-113$.

64. Desquilbet L, Goujard C, Rouzioux C, et al. The Primo and Seroco study groups. Does transient HAART during primary infection lower the virological set-point ? AIDS $2004 ; 18: 2361-9$.

65. Seng R, Goujard C, Desquilbet L, et al. The Primo and Seroco study groups. Rapid CD4+ cell decrease after transient CART initiated during Primary HIV Infection (Anrs-Primo \& Seroco cohorts). Manuscrit en revue.

66. Lanoy $\mathrm{E}$, Mary-Krause $\mathrm{M}$, Tattevin $\mathrm{P}$, et al. Frequency, determinants and consequences to care for HIV infection in France. Antiviral Therapy 2007 ; $12: 89-96$.

67. Lièvre L, Deveau C, Gerbe J, Enel P, Tran L, de Castro N, Costagliola D, Meyer L. Yearly number of patients diagnosed with HIV-1 infection in France ; estimation based on a capture-recapture approach. AIDS 2006 ; $20: 2392-5$. 